\title{
The Atomic and Electronic Structure of the Dynamically Formed $\mathrm{Cu} / \mathrm{Cu}_{2} \mathrm{O}$ Interface Structure
}

\author{
Xuetian Han, Judith C. Yang
}

Department of Materials Science and Engineering, 848 Benedum Hall, University of Pittsburgh, Pittsburgh, PA 15261.

Classical theories [1] of oxidation assume uniform film growth with migrating ions, defects and charged species. This description misses the essential chemical and electronic details of how the oxygen and metal atom convert to a metal-oxide. To understand the kinetics of this transformation requires examining the dynamically formed metal/oxide interface at the nanometer scale and below.

Copper is selected as the model metal system. We have extensive previous work on initial stage of $\mathrm{Cu}$ oxidation by in situ ultra-high vacuum transmission electron microscopy UHV-TEM [2]. Past research on the initial stages of oxidation revealed that the predominant mechanism of the initial transport, nucleation and growth of the oxide is oxygen surface diffusion[3,4], but the details of how the metal and oxygen atoms convert to oxide along the interface is missing from this simplistic description. Also, the precise description of the interface will be directly correlated with electronic structure (ES) theoretical modeling of the interface, where the correct ES description of the interface will provide interface energies and the dynamic charge distribution across the interface needed for the potentials that will be used in molecular dynamic (MD) simulations of oxidation. Experimental determined interface structure is the critical first step of the theoretical model of oxidation mechanisms.

In this study, the $\mathrm{Cu}$ films used to develop $\mathrm{Cu}_{2} \mathrm{O}$ were produced by e-beam evaporation $99.999 \% \mathrm{Cu}$ pellets on irradiated $\mathrm{SrTiO}_{3}$ (100) surface. The $\mathrm{Cu}$ (100) film is about $5000 \AA$ in thickness. The oxide island was formed by in situ oxidation of single crystal $\mathrm{Cu}(100)$ thin films, shown in Figure 1 . The $\mathrm{Cu} / \mathrm{Cu}_{2} \mathrm{O}$ interface was created by oxidation of $\mathrm{Cu}(100)$ film at $\mathrm{P}\left(\mathrm{O}_{2}\right)=8 \times 10^{-4}$ torr for 20 minutes at $350^{\circ} \mathrm{C}$. A dual-beam focused ion beam (FIB) instrument was utilized to select an oxide island, slice through it, and thin the interface. High resolution TEM (HREM) observations shown in Figure 2 revealed an interfacial zone with a thickness approximate $3 \mathrm{~nm}$. Further HREM analysis showed that the distance between the $\mathrm{Cu}_{2} \mathrm{O}\{110\}$ planes decreases with increasing distance from the interface while the $\mathrm{Cu}_{2} \mathrm{O}\{100\}$ plane-spacing increases with increasing distance from the interface. Initial EELS investigations revealed an interfacial region of 3-6nm thickness where the oxidation states changed from $\mathrm{Cu}$ to $\mathrm{Cu}^{+1}$, suggestive of metastable $\mathrm{Cu}$ oxides forming along the interface.

References

[1] N. Birks, G.H. Meier, and F.S. Pettit, Introduction to the high temperature oxidation of metals. Cambridge. 2006

[2] G. Zhou, and J.C. Yang, Applied Surface Science, 210, 165 (2003). 
[3] Yang, J.C., M.D. Bharadwaj, G.W. Zhou, and L. Tropia, Microscopy and Microanalysis, 7(6), 486(2001).

[4] Yang, J.C., D. Evan, and L. Tropia, Applied physics letters, 81(2), 241(2002).

[5] This research project is funded by the Department of Energy (\#DOE FG02 01ER45919) and National Science Foundation (NSF \# 0523205)

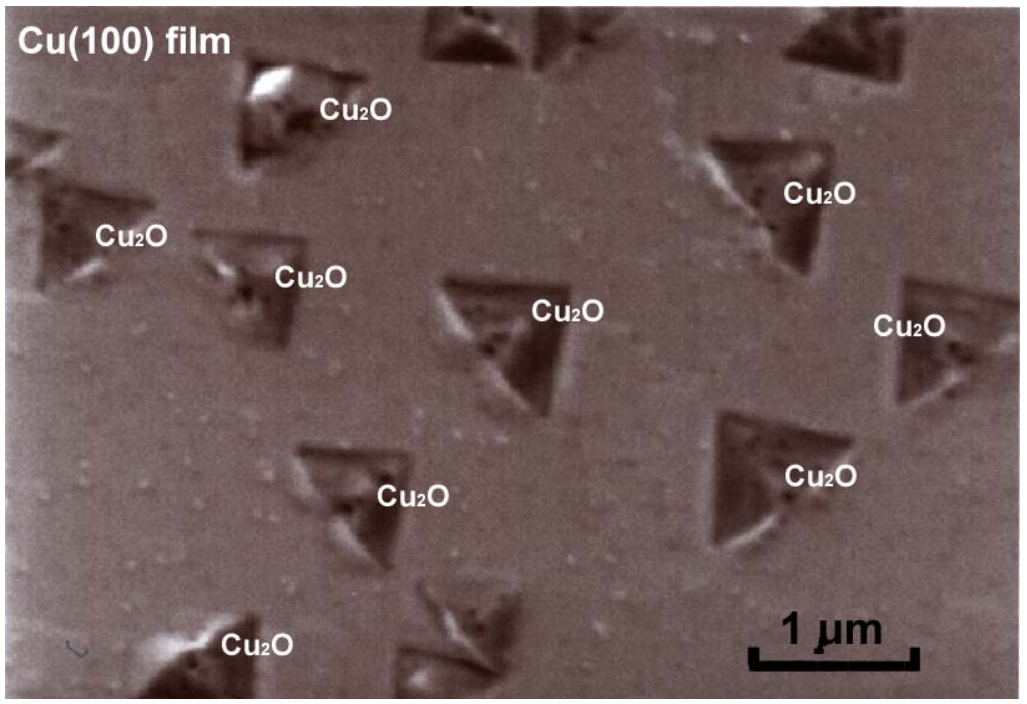

FIG. 1. $\mathrm{Cu}_{2} \mathrm{O}$ triangle islands are formed by oxidation of $\mathrm{Cu}(100)$ film at $\mathrm{P}\left(\mathrm{O}_{2}\right)=8 \times 10^{-4}$ torr for 20 minutes at $350^{\circ} \mathrm{C}$.

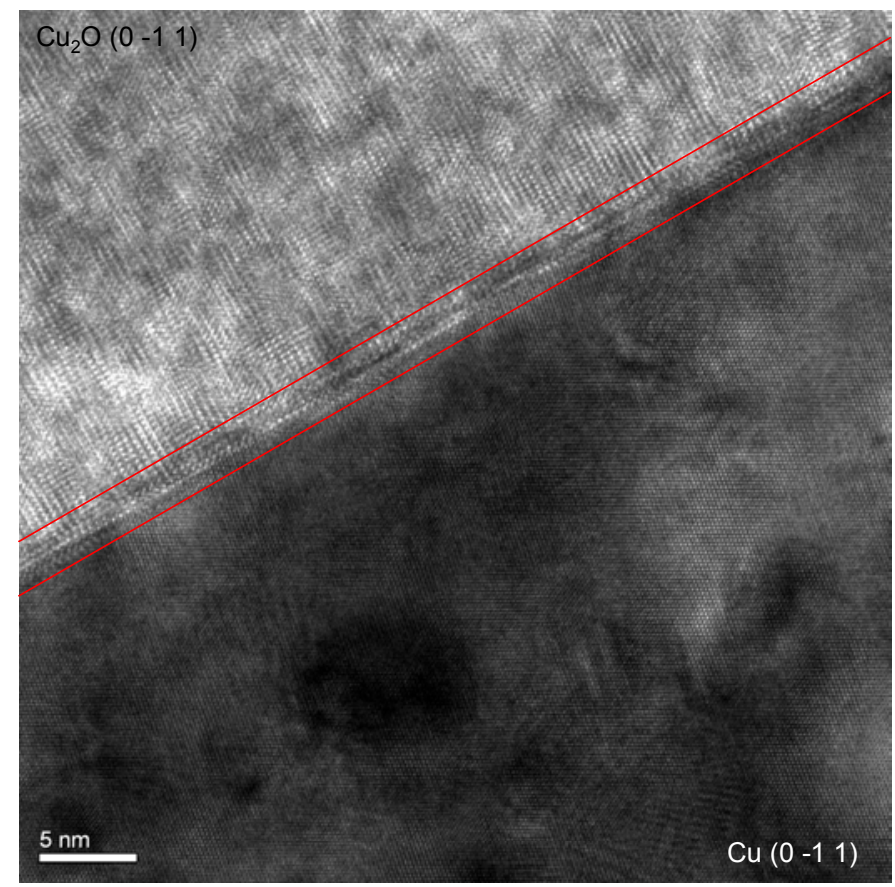

FIG. 2. HREM on the $\mathrm{Cu}_{2} \mathrm{O} / \mathrm{Cu}$ interface structure. The interfacial zone is about $3 \mathrm{~nm}$ thick as indicated by the parallel red lines. 